\title{
La Unión Europea y el Tratado de Lisboa: ¿Posibilidades de una nueva dinámica interna con implicancias externas?
}

\author{
The European Union and the Treaty of Lisbon. Prospects \\ of a new internal dynamics with external implications?
}

\author{
Amalia Stuhldreher ${ }^{*}$
}

Resumen

El artículo centra la atención en algunos de los cambios que conllevará el Tratado de Lisboa para la Unión Europea (UE), en particular respecto de la política externa comunitaria. Se examinan algunos de los modos 'tradicionales' de relacionamiento de la UE con América Latina, así como el nuevo enfoque europeo de vinculación, con la selección de Brasil como socio estratégico. Finalmente se comenta la posible evolución de los vínculos interregionales con vistas a la Cumbre de Lima que tendrá lugar en mayo de 2008.

Palabras Clave: Unión Europea, política externa comunitaria, América Latina, Brasil, cooperación.

\section{Abstract}

The article focuses on some changes that the Lisbon Treaty will imply for the European Union (EU), particularly those related to its common

Profesora, Universidad de Friburgo (astuhldreher@hotmail.com).

Recibido el 26 de octubre de 2007; aceptado el 17 de diciembre de 2007. 
foreign policy. It examines some of the 'traditional' ways of conducting EU's relations with Latin America, as well as the new European approach, which includes the choice of Brazil as a strategic partner. Finally, some comments are made concerning the likely evolution of interregional ties with a view to the Lima Summit, to be held in May 2008.

KEY WORDs: European Union, common foreign policy, Latin America, Brazil, cooperation. 
Amalia Stuhldreher • La Unión Europea y el Tratado de Lisboa...

\section{INTRODUCCIÓN}

El fin de la presidencia pro tempore alemana al frente del Consejo de la Unión Europea (UE) a mediados de 2007 se vio coronado por el acuerdo básico sobre el Tratado de Reforma que marcará el derrotero futuro del bloque ${ }^{1}$. Tras la Cumbre de Lisboa de fines de octubre, el título provisorio de 'Tratado de Reforma' fue modificado por el Tratado de Lisboa, cuyos textos definitivos serían firmados el 13 de diciembre de 2007. Por el momento, el compromiso alcanzado acerca del texto sobre la reforma del Tratado de la Unión permite aplacar las diferencias respecto a las visiones sobre el futuro de Europa y congelar provisionalmente los enfrentamientos ya clásicos entre los federalistas y los defensores de las soberanías nacionales. Resultan claves aquí el impulso que se intenta otorgar a la política exterior común y la posibilidad que se abre para flexibilizar aún más el proceso de integración permitiendo diferentes velocidades en diversas áreas de política.

1 Dicho acuerdo contendrá dos cláusulas substantivas que modificarán, respectivamente, el Tratado de la Unión Europea (TUE) y el Tratado Constitutivo de la Comunidad Europea (TCE). El TUE conservará su denominación actual, mientras que el TCE pasará a llamarse Tratado sobre el funcionamiento de la Unión, ya que la Unión tendrá una única personalidad jurídica. La palabra «Comunidad» se sustituirá en el texto por "Unión»; se estipulará que ambos Tratados son el fundamento de la Unión y que la Unión sustituye y sucede a la Comunidad.
A la luz de los acontecimientos recientes, el presente artículo tematiza en primer término algunas de las modificaciones que incluirá el Tratado de Lisboa, señalando los cambios que traerá aparejados para la política externa comunitaria. En segundo lugar, y a la luz de la evolución registrada en dicho ámbito, se discuten algunos de los mecanismos 'tradicionales' del relacionamiento de la UE con el subcontinente latinoamericano, pasando a focalizar en la tercera parte la nueva estrategia de vinculación de la UE que paulatinamente se hace evidente de cara a América Latina, con la selección de Brasil como socio estratégico. Finalmente se extraen algunas conclusiones y se esbozan perspectivas respecto de la posible evolución de los vínculos interregionales con vistas a la Cumbre de Lima, que tendrá lugar en mayo de 2008.

\section{El COMPROMISO EN TORNO AL Tratado DE Reforma}

El ritmo de avance del proyecto integrativo europeo se vio claramente afectado tras la incorporación de diez países (Chipre, Eslovaquia, Eslovenia, Estonia, Hungría, Letonia, Lituania, Malta, Polonia y República Checa) durante la última rueda de ampliación de mayo de 2004: las profundas dificultades de la ejecución de su ampliación geográfica parecieron consumir el élan vital de la visión de la Europa mancomunada. A esto se sumaría durante los 
dos últimos años la parálisis impuesta por el resultado negativo de los respectivos referendo en Francia y en los Países Bajos sobre la Constitución Europea realizados en $2005^{2}$.

En ese sentido, el comienzo del año 2007 estuvo señalado por las expectativas respecto de la presidencia alemana del Consejo de la UE para superar desafíos que la prensa europea no dejó de comparar con una 'misión imposible' (Kohler 2006), no ya en versión cinematográfica sino de alta política internacional. Su protagonista central, la canciller alemana Angela Merkel, debía asumir la tarea de dinamizar nuevamente un proyecto que había recibido a la vez adhesiones y rechazos y que reflejaba las claras disidencias de las voluntades tanto de parlamentos como de la población afectados.

A pesar de su intención inicial de mantener en carrera el camino de la Constitución, Merkel debió inclinarse ante el peso de las circunstancias y optar por el método clásico de la revisión

2 La asíllamada «Constitución Europea» fue firmada en Roma por los jefes de Estado y de gobierno el 29 de octubre de 2004, y preveía entre otras cosas, la creación de un cargo de Ministro de Asuntos Exteriores (art. I-28 del Tratado Constitucional), que se ocuparía de dirigir la Política Externa y de Seguridad Común (PESC), el «segundo pilar» de la Unión Europea, de orden intergubernamental. Asimismo, debería hacerse cargo de la política europea de seguridad y defensa (PESD). La Constitución contemplaba también la creación del Servicio Europeo de Acción Exterior, encargado de prestar asistencia al Ministro de Asuntos Exteriores. de tratados, renunciando con ello a dotar a la UE de una Constitución, pero posibilitando que se mantuviera la mayor parte de la substancia y esencia del texto constitucional. En consecuencia, a futuro en la UE no habrá bandera, ni himno, ni un texto legal que derogue a los demás y que represente la unidad de Europa. Por otra parte, bajo la presidencia alemana se definieron nuevas reglas para la toma de decisiones, abriendo la posibilidad de darle mayor dinamismo al acordado en Niza (2001), dado que en el futuro los acuerdos en materias tales como emigración, energía, Justicia o Interior podrán tomarse por mayoría cualificada, en lugar de unanimidad $^{3}$ : De acuerdo con el nuevo sistema de votación por doble mayoría, la mayoría necesaria se alcanzaría cuando la decisión en cuestión sea votada por el $55 \%$ de los Estados miembros, que a a su vez representen el $65 \%$ de la UE. A partir de la insistencia polaca se acordó postergar la entrada en vigencia de dicho sistema hasta el año 2014,

3 El Tratado de Niza, firmado en 2001 tras el Consejo Europeo celebrado en dicha ciudad en diciembre de 2000, otorga 29 votos a los países con mayor población como Alemania, Francia, Inglaterra e Italia, mientras que 27 a Polonia, cuya población es mucho menor (Comisión Europea 2001: 11). Los intentos polacos de resistir la modificación de dicho sistema durante la Cumbre de Bruselas a mediados de 2007, llegaron a incluir el argumento de la población muerta durante la Segunda Guerra Mundial, pérdida que esta línea argumentativa atribuye en última instancia a la responsabilidad alemana por el inicio de las hostilidades. 
en vez de 2009 como estaba previsto. Asimismo, en casos conflictivos, hasta 2017 se podrá votar de acuerdo con las reglas de Niza.

En lo referente al rol que le cabe a Comisión de la UE, se decidió reducir el número de sus miembros, de modo que hasta 2014 sus comisarios deberán corresponder a los dos tercios del número de Estados miembros de la UE. Asimismo, el presidente de la Comisión será electo por el Parlamento Europeo teniendo en cuenta los resultados de las elecciones para dicho cuerpo y de acuerdo con la propuesta de los gobiernos europeos. Por su parte, el Parlamento Europeo deberá pronunciarse al mismo nivel que el Consejo de Ministros acerca del presupuesto de la UE, lo que también es válido para amplios aspectos de política interna, sobre la cual los Estados ya no deberán tomar las decisiones en forma unánime, sino también por mayoría.

Por su lado, la Carta de Derechos Fundamentales -a instancia de Inglatera- ya no se convertirá oficialmente en un componente del tratado reformado, aunque una mención en el capítulo de los artículos sobre los derechos fundamentales le otorgará carácter vinculante, con excepción del territorio de Gran Bretaña.

En materia específica de relacionamiento externo de la UE, cabe mencionar que los negociadores ingleses consiguieron debilitar la figura institucional que deberá encargarse de la representación externa de la UE, boicoteando la intención original de crear un Ministro Europeo de Relaciones Exteriores. El acuerdo fue posible a partir de la propuesta española sobre el nombre y competencias de la nueva investidura -que finalmente obtuvo la aceptación británica: el Alto Representante de la UE para Política Exterior y de Seguridad Común deberá asumir las funciones del Alto Comisionado y del Comisario de Comercio Exterior, y será a la vez vicepresidente de la Comisión de la UE. Solo tendrá el derecho indirecto de iniciativa. Asimismo, el Tratado de Lisboa mencionará explícitamente que no se reducirá la competencia de los Estados nacionales en el área de política exterior. Por otra parte, el Consejo Europeo tendrá un presidente permanente que será electo por mayoría cualificada por los jefes de Estado y de gobierno. Deberá dirigir el Consejo como mínimo durante dos años y medio y como máximo cinco, representando a la UE en el exterior, sin menoscabar las atribuciones del Alto Representante. Finalmente, se creará un nuevo servicio diplomático europeo del que podrán formar parte tanto funcionarios de la UE como de los Estados miembros.

La Declaración de Berlín, de marzo de 2007, formulada al cumplirse los cincuenta años de la firma de los Tratados de Roma, postulaba la necesidad de posicionar nuevamente a la UE sobre cimientos comunes, objetivo que puede considerarse alcanzado en la Cumbre de Bruselas, que coronó la presidencia pro tempore alemana. Por cierto, la evaluación efectuada por los actores involucrados acerca de los lo- 
gros obtenidos, refleja naturalmente los condicionamientos propios. Mientras que para el presidente de la Comisión el acuerdo si bien "no es gran lírica», por lo menos representa «una, prosa bastante buena», según la interpretación de Merkel, permitirá a Europa salir de la parálisis sin dejar a nadie excluido del proceso. Por su lado, su par polaco, Jaroslaw Kaczynski, se mostró satisfecho de que su país hubiera obtenido prácticamente todo lo que se había propuesto. Por el contrario, el premier luxembugués, partidario de compromisos más profundos, no dejó de lamentar el nivel de complejidad que adquirirá el texto del nuevo acuerdo, aunque se lo presentara como «tratado simplificado».

En definitiva, los contenidos acordados terminaron de formularse en el marco de la conferencia gubernamental que tuvo lugar bajo la presidencia portuguesa a fines de octubre de 2007. $\mathrm{Al}$ respecto, el premier portugués Sócrates impulsó un rápido cierre de las negociaciones: los textos definitvos del ahora denominado Tratado de Lisboa serían firmados el 13 de diciembre de 2007 en dicha ciudad. De esta forma, el nuevo tratado podría entrar en vigencia a principios de 2009 y proporcionar un nueva base contractual de cara a las elecciones de ese año para el Parlamento Europeo (Presidency of the European Union 2007a). Más allá de dichas intenciones, la dinámica negociadora que se hizo evidente durante la decisiva Cumbre de Bruselas de mediados de 2007 reveló la presencia de una serie de líderes políticos de la nueva generación, que de alguna manera reactivó la tradicional alianza entre Alemania y Francia, a la que en esta ocasión se sumaron el español José Luis Zapatero, Tony Blair, en acuerdo con su designado sucesor Gordon Brown, pero también el luxemburgués Juncker o el lituano Adamkus (Carbajosa 2007; Stabenow 2007). Por otra parte, a fines de la presidencia alemana del Consejo, Eurobarómetro daba cuenta del mayor nivel de aceptación de la UE por los ciudadanos europeos desde 1994: 57\% de la población de los Estados miembros piensa que la participación de su país en la UE constituye un hecho positivo (Eurobarometer, 2007). ${ }^{4}$

\section{El POSICIONAMIENTO DE LA UE FRENTE AL CONTINENTE LATINOAMERICANO: DE LOS MECANISMOS EXISTENTES Y SUS DIFICULTADES MANIFIESTAS}

Como resulta evidente, las reformas acordadas en la Cumbre de Bruselas y que fueron plasmadas en el Tratado de Lisboa apuntan por sobre todo a dinamizar la toma de decisiones, entre otras formas mediante las modificaciones del entramado institucional y de la ampliación de la toma de decisiones por mayoría cualificada a nuevos ámbitos de política. En lo tocante al posiciona-

$4 \quad$ El ranking está encabezado por los Países Bajos $(77 \%)$, Irlanda (76\%) y Luxemburgo $(74 \%)$. 
miento común en materia de política externa, se apunta a lograr una mayor coherencia y un fortalecimiento de la presencia (Allen / Smith, 1991) del actor internacional (Sjöstedt, 1977) en que se ha convertido la UE.

No cabe, sin embargo esperar que a corto o mediano plazo las reformas que se introducirán en este acápite afecten de manera radical el relacionamiento del bloque europeo con América Latina. Más allá de los tiempos necesarios para la formulación e implementación de los nuevos mecanismos y figuras institucionales, los ejes rectores de la vinculación europea con el subcontinente latinoamericano ya han experimentado una redefinición de hecho, que no necesariamente ha sido reconocida como tal dadas las connotaciones implícitas en el nuevo curso de acción elegido. En rigor, sobre todo a nivel discursivo se mantienen al mismo tiempo algunos elementos considerados centrales a la relación birregional como la proclamada búsqueda de una «asociación estratégica", que se vio materializada en el proceso de cumbres iniciado en 1999 en Río de Janeiro y los encuentros posteriores de Madrid (2002), Guadalajara (2004) y Viena (2006) y la Cumbre de Lima que debe realizarse en mayo de 2008. En forma paralela, los diálogos con los diferentes esquemas subregionales (Mercosur, CAN y Centroamérica) representan hoy por hoy el recurso retórico privilegiado en las presentaciones oficiales.

Sin embargo, la falta de avances precisos en los respectivos carriles de relacionamiento se ha hecho evidente desde hace tiempo. Al respecto, resulta paradigmático el dificultoso devenir del proceso de negociación entre la UE y el Mercosur para la constitución de una «asociación interregional de carácter político y económico», en especial debido a la imposibilidad de cerrar el capítulo de la negociación comercial. Por su parte, la última visita del director de Comercio de la Comisión Europea, Karl Falkenberg, a los países del Mercosur pretendió señalar que a la Europa unificada aún le interesa un acuerdo comercial entre los bloques. En forma correspondiente, el último párrafo de la declaración conjunta emitida durante la última Cumbre de Asunción de los jefes de Estado del Mercosur, celebrada en junio de 2007, indica en forma casi rutinaria la importancia de retomar a la brevedad las negociaciones birregionales con los socios europeos.

Por su parte, tras la Cumbre de Viena de mayo de 2006 los procesos de negociación con la CAN y con Centroamérica han registrado algunos avances. Así, tras la aprobación de las directrices de negociación por el Consejo de la UE en abril de 2007 (Council of the European Union Press Release 23-24/04/2007: 35), a mediados de junio de 2007 se anunció solemnemente el inicio de las negociaciones entre la CAN y la UE por un Acuerdo de Asociación integral (Acta de Tarija 2007; EU Press Releases 14/06/2007). En lo que hace a la cooperación a futuro, y siguiendo el esquema existente, el Documento de Estrategia Regional de la Comisión Eu- 
ropea para la CAN 2007-2013 señala que la integración es el gran objetivo para la región andina: así, en los sectores seleccionados para la cooperación se apunta en primer término al apoyo a la integración económica andina para lograr un mejor posicionamiento en el escenario internacional (Comisión Europea 2007a: 1$)^{5}$.

Asimismo, el Documento de Estrategia Regional de la Comisión Europea para América Central para el período 2007-2013 también identifica la integración regional como prioridad central para la cooperación (Comisión Europea 2007b: 1$)^{6}$. En forma concordante, a fines de junio se dio a conocer el comienzo de las negociaciones con Centroamérica que apuntan también a un acuerdo de asociación, que incluirá a Panamá (EU Press Releases 29/06/07). Sin embargo, teniendo en cuenta la dilatación temporal de ambas inicia-

$5 \quad$ Para el período 2007-2013 se destina una asignación indicativa de 50 millones de euros para la CAN mediante el mecanismo financiero de cooperación para el desarrollo (ICD). Se indica asimismo que estos recursos pueden complementarse con proyectos y programas financiados mediante programas bilaterales para cada país de la CAN y mediante el programa regional latinoamericano, así como con programas temáticos.

6 Para el período 2007-2013 se destina una asignación indicativa de 75 millones de euros para Centroamérica, con arreglo al mecanismo financiero de cooperación para el desarrollo (ICD). Este monto de reforzará a través de los proyectos financiados por las líneas presupuestarias específicas de la CE y por los programas regionales de América Latina. tivas, la situación resulta contrastante con la rapidez con que la UE alcanzó los respectivos acuerdos de asociación con México (2000) y con Chile (2002). Aun así, si se observan las repercusiones concretas de los acuerdos, y más allá de las coincidencias registradas en materia de política exterior, resulta evidente que pese a una mayor dinámica en los intercambios comerciales, ella no significó una modificación realmente sustancial de los patrones ya vigentes.

En otro nivel de relacionamiento birregional, en el último encuentro de los ministros de asuntos exteriores de la UE y sus pares del "Grupo de Río», la paleta de temas discutidos se extendió desde la problemática de Haití, la energía, el medio ambiente y el cambio climático, hasta la consolidación del multilateralismo, los países de ingreso intermedio y su lucha contra la pobreza, para finalizar con la discusión sobre el futuro de dicho foro. En esa ocasión, la UE presentó su nueva estrategia de cooperación para América Latina (2007-2013) que incluirá un paquete de ayuda por un total de 2.690 millones de euros (EU Press Releases 19/04/2007) 7 . Sin embargo, no puede menos que registrarse la pérdida de peso y el desdibujamiento del perfil del Grupo de Río.

\footnotetext{
Los temas señalados como prioritarios forman desde hace tiempo el repertorio de tópicos privilegiados por la UE: fomento de la cohesión social, apoyo a la integración regional y la cooperación económica, promoción del conocimeinto mutuo entre la UE y América Latina, gobernabilidad y derechos humanos, así como desarrollo sostenible.
} 
Dada su creciente inoperancia de cara a un posicionamiento externo conjunto latinoamericano y considerando su incapacidad de convertirse en un mecanismo de seguimiento de los avances durante el tiempo transcurrido entre las cumbres birregionales, desde la comunidad científica europea se han dado pronunciamientos críticos que señalan incluso la necesidad de discontinuar la cooperación birregional en el marco de dicho foro (Maihold, 2006: 16).

\section{Europa-AmÉrica Latina: ¿HACIA UN NUEVO TIPO DE VINCULACIÓN?}

Así pues, se hace evidente que más allá de la continuación de los procesos mencionados, la UE comenzó ya hace tiempo a vislumbrar otra forma de vinculación que sería en cierta forma más funcional a sus necesidades estratégicas de posicionamiento en el escenario global. Sin descartar formalmente su rol de «federador externo» frente a los procesos integrativos en América Latina, y frente a demandas concretas de coordinación que imponen la definición de mecanismos de diálogo para conciliar posiciones en áreas como las negociaciones de la OMC, los debates de las Naciones Unidas o la compleja situación política en América Latina, la UE ha introducido una nueva lógica de relacionamiento mediante la nueva estrategia de la Comisión Europea frente a América Latina, hecha pública en diciembre de 2005 (Comisión Europea
2005), que subraya la necesidad de atender en forma particular las relaciones con los países más grandes de la región, como México, pero en forma especial Brasil ${ }^{8}$.

En consecuencia, a los mecanismos de relacionamiento ya vigentes se suma un nuevo vector de vinculación, que actualmente materializa en la propuesta de llevar a Brasil al nivel de socio estratégico, estatus del que ya gozan Estados Unidos, China, Japón, India y Sudáfrica y que también pretende Rusia. Este nuevo tipo de vinculación privilegiada con Brasil, que se refleja en el Documento de Estrategia para Brasil (European Commission Europea 2007c), se selló en la Cumbre UE-Brasil en Lisboa a comienzos de julio de 2007, donde se acordó también definir un Plan de Acción conjunto para la implementación de los objetivos postulados. Por sobre todo, y desde el punto del posicionamiento externo de la UE y del Brasil como actores internacionales, resulta interesante que en sus declaraciones se coincida en cuanto al rol atribuido al 'multilateralismo efectivo' con las

8 Respecto de Brasil, el documento señala que por el momento la UE sólo dispone de estructuras de diálogo exiguas, carentes de dimensión política. Esto ya no reflejaría la realidad de la rápida evolución de Brasil como polo ecóomico y político mundial. Asimismo, a Brasil le correspondería un papel central en la integración regional, objetivo central de la cooperación de la UE. En cuanto a México, se subraya la necesidad de seguir el trabajo emprendido para optimizar el potencial que ofrece el acuerdo de asociación (Comisión Europea 2005: 18). 
Naciones Unidas como factor central, así como en la postulada intención de actuar en forma conjunta para hacer frente a desafíos globales tales como la paz y la seguridad mundiales, la democracia y los derechos humanos, el cambio climático, la biodiversidad, la seguridad energética y el desarrollo sustentable9 , así como la lucha contra la pobreza y la exclusión (Presidency of the European Union, 2007).

Oficialmente, la propuesta se presenta como inserta en los lineamientos vigentes para la asociación birregional. Sin embargo, no puede dejar de advertirse que la selección de un país en particular, al que se atribuye el carácter de "polo económico y político mundial» (Comisión Europea 2005: 18), representa una clara modificación de los mecanismos hasta ahora vigentes frente a América Latina. Se reconoce así el rol desempeñado por Brasil en foros claves, tales como el G4, que resultan centrales para las negociaciones comerciales internacionales. En dichas instancias, Brasil actúa como Estado nacional sin mandato vinculante para representar

9 Coincidiendo con el lanzamiento de la asociación estratégica UE-Brasil, en el marco de su política externa energética la UE organizó una conferencia internacional sobre biocombustibles que convocó a decisores gubernamentales, organizaciones no gubernamentales, académicos y representantes del sector privado, a fin de discutir el desarrollo de un enfoque internacional para la producción, uso y comercialización de estos combustibles, con el objetivo de reforzar la seguridad enegética y reducir las emisiones de dióxido de carbono (EU Press Releases 3/07/2007). a sus socios del Mercosur. $\mathrm{Al}$ respecto, algunos analistas críticos han planteado por un lado la necesidad implícita de consultas previas -aun teniendo en cuenta el carácter informal de las reuniones del G4- si se quiere preservar el espíritu original del bloque (Peña 2007). A la luz de los acontecimientos recientes cabe sin embargo señalar que pese a actuar en función de sus intereses globales, los esfuerzos emprendidos por la diplomacia de Brasil para encaminar esfuerzos colectivos hacia el fortalecimiento del poder de presión de los países en vías de desarrollo frente a las potencias industrializadas mediante la creación del G20, nunca pudieron prescindir de su base regional. Así, Brasil ha debido buscar un equilibrio entre sus nuevos socios políticos, como la India, y vínculos prioritarios como su relación con Argentina (Hirst 2007). Aun pese a la ausencia de una coordinación profunda de posiciones con sus pares del Mercosur de cara al G4, tras el fracaso del encuentro de Potsdam que apuntaba a destrabar las negociaciones de la OMC, el gobierno argentino efectuó una encendida defensa de la actuación brasileña en dicho foro (Newsletter Mercosur ABC. 28/06/2007).

Otro de los argumentos críticos de la decisión europea de designar a Brasil como socio estratégico subraya el posible debilitamiento implícito de dicha estrategia para el Mercosur en su calidad de elemento clave para la estabilidad política y la integración en el Cono Sur (Peña, 2007). De hecho, la concreción de la propuesta europea 
a Brasil se da en momentos en que se ponen de manifiesto las diferentes concepciones existentes dentro de los miembros del Mercosur respecto de la proyección y esencia del proceso integrativo, en especial por el enfrentamiento entre la visión brasileña y la visión del presidente Hugo Chávez, que postula con dialéctica maniquea la oposición entre el «viejo»y el «nuevo» Mercosur ${ }^{10}$.

Para algunos observadores del proceso, la tendencia observada en el curso de acción europeo señala el quiebre de la Europa unificada con el esquema «autoimpuesto» del interregionalismo ${ }^{11}$

10 Tras su ausencia de la cumbre de jefes de Estado del Mercosur celebrada en Asunción a fines de junio de 2007, el presidente Chávez se mostró incluso dispuesto a retirar la solicitud de adhesión plena al bloque si este se limitaba a ser un mero mecanismo «signado por el capitalismo y la competencia feroz». Como consecuencia de la polémica generada con el Senado de Brasil tras su reclamo por la no renovación de licencia en un canal de televisión privado en Venezuela por parte de Chávez, y a la espera de una disculpa por los dichos del presidente venezolano, dicho cuerpo no ha otorgado todavía la aprobación parlamentaria para el ingreso de Venezuela al bloque.

11 Para una consideración teórica del concepto de interregionalismo véase Rüland 2005: 298. Resumiendo la discusión, el autor propone utilizar el concepto como un término genérico que abarcará todo el espectro de formatos creados por las regiones para llevar adelante su interacción externa. Así, el «interregionalismo» podría subdividirse en a) «birregionalismo» o «interregionalismo bilateral», lo que abarcaría los diálogos de grupo a grupo, y b) «transregionalismo», de acuerdo con y una tardía aceptación del hecho de que el diálogo político con Brasil también puede tener lugar al exterior del Mercosur. En ese sentido, debido entre otras cosas a las fuerzas centrífugas presentes en América Latina, actualmente se haría evidente el reemplazo del marco del interregionalismo por una nueva forma de relacionamiento cuya denominación abarcaría denominaciones diversas, que irían desde 'interregionalismo híbrido' (Söderbaum / van Langenhove 2005) hasta un 'bilateralismo múltiple', y en que donde diversos actores a ambos lados del Atlántico buscarían imponer sus propios intereses. De esta forma se relativizarían acuerdos e instancias interregionales favorables a estructuras variables en la que los distintos intereses de los socios podrían lograr una mejor coordinación. Así, disminuiría la inclinación de la UE a forzar la «construcción» de una contraparte por medio del fomento de los procesos de integración subregionales, que ya tampoco son apoyados a rajatabla dentro del mismo subcontinente latinoamericano (Maihold 2006: 9 y ss.).

\section{CONCLUSIONES Y PERSPECTIVAS}

Las modificaciones que traerá aparejado el Tratado de Lisboa a la arquitectura de la Europa unificada apuntarán a la agilización de los proce-

el cual los miembros actúan usualmente a título individual y no necesariamente están presentes todos los miembros de una organización regional determinada. 
sos decisorios dentro de un entramado institucional fortalecido. Entre otras cosas, se intenta a partir de ello lograr una presencia más asertiva de la UE en el escenario internacional. Sin embargo, los cambios previstos no serán factores de peso que modifiquen de por sí la vinculación de la UE con el subcontinente latinoamericano. Más allá de que el Alto Representante de la UE para Política Exterior y de Seguridad Común deberá asumir las funciones del actual Alto Comisionado así como las del actual Comisario de Comercio Exterior, siendo a la vez el vicepresidente de la Comisión de la UE, cabe esperar que las diferencias existentes entre la sólida coordinación de la política exterior comercial dentro del así llamado "primer pilar» de la UE y la dificultosa concertación de posicionamientos conjuntos en materia de política exterior dentro del «segundo pilar» no serán superadas rápidamente . Para las contrapartes, incluidos los partners latinoamericanos, esto significará, entre otras cosas, que en el futuro cercano también deberá contarse con firmes posiciones europeas en materia de negociaciones comerciales internacionales, que muy probablemente continuarán colisionando con los intereses latinoamericanos, opuestos al proteccionismo agrícola comunitario.

En lo que hace al diálogo políticodiplomático, cabe esperar a futuro que la jerarquización de la figura del representante europeo pueda contribuir al afianzamiento de su posición dentro de las instancias de decisión europeas, aunque no puede excluirse la posibilidad de cierta competencia con el presidente del Consejo de la UE en lo tocante la representación externa de Europa. De cara a América Latina y a nivel de protagonistas, es posible que se profundice la tendencia ya señalada a identificar países centrales, estrategia que frente a la necesidad de racionalizar el propio rol, presumiblemente seguirá presentándose como parte de las líneas ya vigentes, pero que en definitiva responde a motivaciones diferentes, condicionadas por las particulares necesidades de posicionamiento de la UE en el escenario internacional. En cuanto al contenido del diálogo con los partners latinoamericanos, posiblemente los temas de índole eminentemente política seguirán mezclándose parcialmente con cuestiones de corte más cercano a la asistencia para el desarrollo. Dicha evolución, que se ha hecho evidente en los últimos años, implica un desdibujamiento de los respectivos niveles de interacción contra el cual representantes de la comunidad académica ya han formulado advertencias. Asimismo, han señalado que es apremiante definir una nueva base para el relacionamiento UE-América Latina, superando la mera la invocación ritual de la historia y la herencia cultural compartida. Al respecto, se han esbozado propuestas para redefinir el formato de las relaciones (en especial el de la diplomacia de cumbres, incluido el fortalecimiento de la dimensión ejecutiva de dicho proceso), los contenidos y coherencia de la agenda interregional, lo que incluye la revisión de la integración como modelo 
Amalia Stuhldreher • La Unión Europea y el Tratado de Lisboa...

rector para América Latina (Maihold 2006: 14 y ss.).

Al momento de examinar y realizar una reflexión prospectiva que permita definir nuevas perspectivas de relación interregional de cara a la próxima Cumbre que debe celebrarse en Lima en mayo de 2008, diversos análisis otorgan un lugar central a la inclusión activa de la sociedad civil en la agenda interregional, factor que entre otras cosas otorgaría mayor legitimidad y dinamismo a los procesos de negociación en curso (Peña 2006: 13 y 18; Maihold 2006: 18ss.). Al repecto, para superar el carácter simbólico de dicha participación se sugiere, entre otras cosas, adoptar el modelo de discusión utilizado para la propuesta constitucional europea, lo que permitiría analizar y discutir propuestas temáticas concretas en períodos claramente definidos entre cumbre y cumbre (Maihold 2006: 18)

Sin lugar a dudas un avance decidido en esa dirección tendría la ventaja de otorgar un valor agregado particular a los vínculos interregionales, lo que funcionaría a la vez como reaseguro y fuente de diversificación de estos, por mucho que no pueden negarse las dificultades que ya han quedado de manifiesto en esta materia, entre otras razones por la debilidad de los representantes de la sociedad civil o por los intentos de cooptación proveniente de los sectores gubernamentales pertinentes. Sin embargo, siendo dicha integración genuina una condición necesaria para superar el impasse de la relación interregional, resulta evidente que no basta para lo- grar dicho objetivo. Al respecto, cabe señalar que la sociedad civil difícilmente podrá abarcar o compensar la falta de liderazgo político en América Latina que disponga de claridad de metas frente a los socios europeos y que resultaría necesario para definir una hoja de ruta operativa para Lima 2008.

Dadas las conocidas dificultades en lo tocante a la concreción de posicionamientos externos conjuntos y teniendo en cuenta las actuales tendencias centrífugas en el Cono Sur, de momento no cabe esperar que la reversión del letargo de que adolece el vínculo interregional tenga como protagonista central al vector latinoamericano. Sin embargo, el fracaso de la reunión del G4 en Potsdam incluye elementos interesantes de consideración. Por un lado, la opción multilateral de liberalización comercial pareciera por el momento cada vez más inalcanzable y podría conducir a una revalorización del camino interregional, que carga sin embargo con un lastre significativo de demoras y desacuerdos; por la otra, el rol de la diplomacia brasileña en el G4 -incluyendo en su accionar la perspectiva subregional- , sumado a su aceptación más o menos implícita por los miembros fundadores del Mercosur -en especial Argentina(Newsletter ABC. 5/07/2007), que tampoco explicitaron críticas directas a la decisión europea de privilegiar a uno de los socios, implicaría un punto de partida para el necesario fortalecimiento de la concertación.

Los desafíos al respecto no son menores, dado que la inclusión de Ve- 
nezuela ha hecho aún más compleja la definición de posiciones conjuntas. Sin embargo, reconociendo la imposibilidad inmediata de modificar las nuevas reglas del juego introducidas por la UE, los socios del Mercosur deberían hacerse cargo de su propia debilidad, procurando sacar provecho de su inclusión indirecta en la "carta brasileña» a partir de su participación en el bloque 'liderado' por Brasil. A la inversa, las autoridades brasileñas parecen reconocer que pese al peso propio de Brasil en el escenario internacional por su condición como potencia intermedia, su atractivo como partner disminuiría si se desvinculara del ámbito sudamericano que, por otra parte, constituye su espacio natural de proyección.

\section{Bibliografía}

Acta de Tarija (2007), Inicio de las negociaciones para un Acuerdo de Asociación entre la Comunidad Andina y la Unión Europea, 14 de junio.

Allen, David y Michael Smith (1991), Western Europe's Presence in the Contemporary International Arena, en: Holland, Martin (ed.), The Future of European Political Cooperation. Essays on Theory and Practice. Londres, Macmillan. Houndsmills (pp. 95-120).

Carbajosa, Ana (2007), Los nuevos estadistas redefinen las alianzas en Europa, en «El País», Madrid, 25 de junio.

Comisión Europea (2001), ¿Cuál es la contribución del Tratado de Niza? Instituciones y órganos en la Unión Europea. ¿Quién hace qué en la Unión Europea?, Luxemburgo.

Comisión Europea (2005), Una asociación reforzada entre la Unión Europea y América
Latina, Comunicación de la Comisión al Consejo y al Parlamento Europeo.

Comisión Europea (2007ª), Comunidad Andina. Documento de Estrategia Regional. 2007-2013. (E/2007/678).

Comisión Europea (2007b), América Central, Documento de Estrategia Regional 2007-2013. (E/2007/481).

Council of the European Union, Comunicado de prensa (2007), 23-24 abril, 2795th/2796th Council meetings. General Affairs and External Relations, Luxemburgo.

EU Press Releases 19/04/2007, Los Ministros de Asuntos Exteriores de la UE y de América Latina se reúnen en Santo Domingo los dias 19 y 20 de abril, IP/07/529.

EU Press Releases 14/06/07, EU and the andean Community launch negotiations for Association Agreement», IP/07/834.

EU Press Releases 29/06/2007, EU and the Central American Community start negotiations for new Association Agreement, IP/07/981.

European Commission Press Releases, 3/07/2007, European Commission gathers key international players to discuss sustainable development of biofuels, IP/07/1000.

Eurobarometer 67, Public Opinion in the European Union. European Commission, junio de 2007.

European Commission (2007c), Brazil. Country Strategy Paper. 2007-2013. (E/2007/889).

Hirst, Mónica (2007), El MERCOSUR, en estado crónicamente precario «, Clarín, 3 de julio de 2007.

Kohler, Berthold (2007), Das polnische Menetekel, en: Frankfurter Allgemeine Zeitung. Frankfurt, 25 de junio de 2007.

Maihold, Günther (2006), Nach dem Wiener Gipfel. Europas schwieirige Suche nach einem besonderen Verhältnis zu Lateinamerika, Ibero-Analysen, Heft 19 de junio 2006.

Newsletter MERCOSUR ABC, 28/0672007, Argentina da su apoyo a posición de Brasil en Ronda Doha. 
Newsletter MERCOSUR ABC, 5/07/2007, XXXIII Cumbre del MERCOSUR. Flexibilización para las pequeñas economías. Politica externa coordinada. El liderazgo de Brasil.

Peña, Félix (2006), Der europäisch-lateinamerikanische Dialog und seine Zukunft: Anmerkungen zur Vorbereitung des Gipfeltreffens in Lima 2008, en: Europa -Lateinamerika: Wege zu einer neuen interregionalen Agenda ", Europa América Latina $\mathrm{N}^{\circ} 22$, Analysen und Berichte, KAS, diciembre, (pp. 13-20).

Peña, Félix (2007), Consistencia del Mercosur en sus frentes externos. Algunos interrogantes, en: Newsletter Mercosur ABC, 24 de mayo de 2007.

Presidency of the European Union (2007a), Press Releases. Federal Foreign Minister Steinmeier: Taking stock of Germany's EU Presidency, 27.de junio de 2007.
Presidency of the European Union (2007b), Joint Statement at the EU-Brazil Summit, 4 de julio de 2007.

Rüland, Jürgen (2005), Interregionalism. An unfinished agenda, en: Rüland, Jürgen, Heiner, Hänggi y Ralf Roloff (eds), Interregionalism and International Relations. Routledge, Nueva York, Abingdon, (pp. 295-313).

Sjöstedt, Gunnar (1977), The External Role of the European Union. Swedish Studies in International Relations, Bd. 7. Westmead.

Söderbaum, Frederik y Luk van Langenhove (2005), Introduction: The EU as a Global Actor and the Role of Interregionalism, en: Journal of European Integration. Vol. 27, (pp. 249-262)

Stabenow, Michael (2007), Der Erfolg des langen Atems, en: Frankfurter Allgemeine Zeitung, 25 de junio de 2007. 